\title{
Stratégies identitaires de jeunes issus de l'immigration et contextes scolaires
}

Vers un renouvellement des figures de la reproduction culturelle Identity Strategies of Young Immigrants and School Contexts Renewing Cultural Reproduction Strategies Estrategias identitarias de jóvenes provenientes de la inmigración y contextos escolares Hacia la actualización de las figuras de la reproducción cultural

\section{Marie Verhoeven}

Volume 34, numéro 1, printemps 2006

La contribution de l'école au processus de construction identitaire des élèves dans une société pluraliste

URI : https://id.erudit.org/iderudit/1079036ar

DOI : https://doi.org/10.7202/1079036ar

\section{Aller au sommaire du numéro}

\section{Éditeur(s)}

Association canadienne d'éducation de langue française

\section{ISSN}

0849-1089 (imprimé)

1916-8659 (numérique)

Découvrir la revue

Citer cet article

Verhoeven, M. (2006). Stratégies identitaires de jeunes issus de l'immigration et contextes scolaires : vers un renouvellement des figures de la reproduction culturelle. Éducation et francophonie, 34(1), 95-110.

https://doi.org/10.7202/1079036ar

\section{Résumé de l'article}

Cet article propose une analyse renouvelée des stratégies de construction identitaire de jeunes (16-18 ans) « issus de l'immigration ", dans le cadre des sociétés plurielles et individualisées. À partir d'entretiens sociobiographiques menés dans des établissements contrastés en Communauté française de Belgique, l'auteur décrypte ces stratégies identitaires dans une double perspective. D'un point de vue synchronique, elle analyse les répertoires identitaires et linguistiques mobilisés, en lien avec les contextes scolaires fréquentés. En contextes défavorisés, les répertoires identitaires oscillent entre deux pôles : un répertoire " essentialisant» et un répertoire " d'hybridation anomique "; les répertoires linguistiques y sont dominés par une logique d'hybridation «non réflexive ». En contextes favorisés, les répertoires identitaires oscillent entre un pôle assimilationniste et diverses stratégies non assimilationnistes (hybridation réflexive, diffraction stratégique...); les répertoires linguistiques s'apparentent à un usage stratégique et réflexif de codes différenciés. La seconde partie de l'article aborde, d'un point de vue diachronique, le rôle de la trajectoire des élèves dans la constitution de ces répertoires. Le poids des attentes normatives des établissements successivement fréquentés, ainsi que le rôle des acteurs scolaires dans l'activation des répertoires, sont mis en évidence. La typologie finale permet de dépasser l'opposition classique entre assimilation et réussite d'une part, parcours scolaires difficiles et " ethnicisation » de l'autre.
Tous droits réservés (C) Association canadienne d'éducation de langue française, 2006
Cecument est protégé par la loi sur le droit d'auteur. L'utilisation des services d'Érudit (y compris la reproduction) est assujettie à sa politique d'utilisation que vous pouvez consulter en ligne.

https://apropos.erudit.org/fr/usagers/politique-dutilisation/ 


\title{
Stratégies identitaires de jeunes issus de l'immigration et contextes scolaires : vers un renouvellement des figures de la reproduction culturelle
}

\author{
Marie VERHOEVEN \\ Université catholique de Louvain, Louvain-la-Neuve, Belgique
}

\section{RÉSUMÉ}

Cet article propose une analyse renouvelée des stratégies de construction identitaire de jeunes (16-18 ans) "issus de l'immigration", dans le cadre des sociétés plurielles et individualisées. À partir d'entretiens sociobiographiques menés dans des établissements contrastés en Communauté française de Belgique, l'auteur décrypte ces stratégies identitaires dans une double perspective. D'un point de vue synchronique, elle analyse les répertoires identitaires et linguistiques mobilisés, en lien avec les contextes scolaires fréquentés. En contextes défavorisés, les répertoires identitaires oscillent entre deux pôles : un répertoire "essentialisant» et un répertoire «d'hybridation anomique »; les répertoires linguistiques y sont dominés par une logique d'hybridation "non réflexive». En contextes favorisés, les répertoires identitaires oscillent entre un pôle assimilationniste et diverses stratégies non assimilationnistes (hybridation réflexive, diffraction stratégique...); les répertoires linguistiques s'apparentent à un usage stratégique et réflexif de codes différenciés. La seconde partie de l'article aborde, d'un point de vue diachronique, le rôle de la 
trajectoire des élèves dans la constitution de ces répertoires. Le poids des attentes normatives des établissements successivement fréquentés, ainsi que le rôle des acteurs scolaires dans l'activation des répertoires, sont mis en évidence.

La typologie finale permet de dépasser l'opposition classique entre assimilation et réussite d'une part, parcours scolaires difficiles et « ethnicisation » de l'autre.

\section{ABSTRACT}

\section{Identity Strategies of Young Immigrants and School Contexts : Renewing Cultural Reproduction Strategies}

Marie Verhoeven

Catholic University of Louvain, Louvain-la-Neuve, Belgium

This article offers a renewed analysis of the identity building strategies of young immigrants (16-18 year-olds) in the context of pluralistic and individualized societies. Basing her analysis on sociobiographical interviews held in contrasting establishments in Belgium's French community, the author decrypts these identity strategies from a dual perspective. From a synchronic point of view, she analyses the mobilized identity and linguistic repertoires in relation to the contexts of the schools attended. In socially disadvantaged contexts, the identity repertoires oscillated between two poles : an " essentializing " repertoire and an " anomic hybridization " repertoire. These linguistic repertoires are dominated by a non-reflexive hybridization logic. In socially-advantaged contexts, the identity repertoires oscillated between an assimilationist pole a variety of non-assimilationist strategies (reflexive hybridization, strategic diffraction...), the linguistic repertoires being part of a strategic and reflexive use of differentiated codes. From a diachronique point of view, the second part of the article tackles the role of the students' trajectory in building these repertoires. The weight of normative expectations of the establishments the students successively attend, as well as the role of school players in the activation of these repertoires are revealed. The final typology goes beyond the classic opposition between assimilation and success, as well as difficult school experiences and « ethnicization». 


\section{RESUMEN}

\section{Estrategias identitarias de jóvenes provenientes de la inmigración y contextos escolares : Hacia la actualización de las figuras de la reproducción cultural}

Marie Verhoeven

Universidad católica de Lovaina, Lovaina-la-Nueva, Bélgica

Este artículo propone un análisis actualizado de las estrategias de construcción identitaria de los jóvenes (16-18 años) «provenientes de la inmigración », en el contexto de sociedades plurales et individualizadas. A partir de entrevistas socio-biográficas realizadas en establecimientos contrastados de la Comunidad francesa de Bélgica, el autor descifra las estrategias identitarias a través de una doble perspectiva. De un punto de vista sincrónico, analiza los repertorios identitarios y lingüísticos movilizados, relacionándolos con los contextos escolares frecuentados. En contextos desfavorecidos, los repertorios identitarios oscilan entre dos polos: un repertorio " esencializante " y un repertorio « de hibridación anómica »; los repertorios lingüísticos se encuentran dominados por una lógica de hibridación "no reflexiva ». En contextos favorecidos, los repertorios identitarios oscilan entre un polo asimilacionista y diversas estrategias no asimilacionistas (hibridación reflexiva, difracción estratégica...); los repertorios lingüísticos se asemejan al uso estratégico y reflexivo de códigos diferenciados. La segunda parte del artículo aborda, desde un punto de vista diacrónico, el rol de la trayectoria de los alumnos en la constitución de dichos repertorios. S ponen en evidencia el peso de las expectativas normativas de los establecimientos frecuentados y el rol de los actores escolares en la activación de dichos repertorios. La tipología final permite rebasar la oposición clásica entre asimilación y éxito por un lado, trayectoria escolar difícil y « etnización » del otro.

\section{Introduction}

L'étude des rapports entre position scolaire et identité socioculturelle constitue depuis toujours l'un des axes de réflexion majeurs de la sociologie de l'éducation (Barrère et Sempel, 1998). En ce qui concerne l'intégration scolaire des jeunes « issus de l'immigration ", les recherches classiques tendent à converger autour d'un modèle central fondé sur l'opposition entre, d'une part, assimilation culturelle et réussite scolaire, et d'autre part, parcours scolaires difficiles et non-assimilation ou « ethnicisation" (maintien ou renforcement des particularismes culturels) (voir par ex. Hammersley Woods, 1973). Si cette lecture semble aujourd'hui encore partiellement de mise, les transformations contemporaines amènent à la renouveler. Nous 
L'identité ne constitue pas un "donné " figé, mais s'apparente à un processus qui se construit et se déconstruit au fil des contextes fréquentés, dans une dynamique entre autocatégorisation et catégorisation par autrui. défendrons ici l'hypothèse que, si cette analyse prenait tout son sens dans le cadre d'États-Nations modernes culturellement homogènes (Gellner, 1994), l'ensemble des phénomènes communément regroupés sous le terme de " globalisation », l'hétérogénéité sociale et culturelle croissante des contextes de socialisation (dont les contextes scolaires), ainsi que la complexification des processus de construction identitaire, transforment radicalement la donne. Au moment où l'image d'un « homme pluriel» (Lahire, 2001) s'impose comme figure dominante de l'individu contemporain, ne faut-il pas se doter de nouveaux outils conceptuels pour saisir la fonction de reproduction culturelle de l'école? Comment se construit, du côté des trajectoires biographiques et scolaires des jeunes, la capacité à "vivre dans plusieurs mondes " (de Singly, 2003)? Quelle est la place des usages linguistiques (langue "nationale » I langues "d'origine ») dans ce processus? C'est à ces questions que cet article entend contribuer, à partir de résultats de recherches ${ }^{1}$ portant sur les stratégies identitaires de jeunes "issus de l'immigration" en Communauté française de Belgique et à Bruxelles. Nous viserons à mettre en évidence l'articulation entre ces stratégies identitaires et les contextes scolaires dans lesquels elles se déploient.

D'un point de vue théorique, nos analyses s'inscrivent dans le sillage des développements contemporains des théories de l'identité sociale et de la socialisation (Dubar, 2000, Lahire, 2001, de Singly, 2003, Verhoeven, 1997/2005), qui délaissent les définitions "essentialistes" au profit de conceptions à la fois plus dynamiques, plurielles et contextualisées de la construction sociale des identités. Il est en effet aujourd'hui largement admis que l'identité ne constitue pas un "donné " figé, mais s'apparente à un processus qui se construit et se déconstruit au fil des contextes fréquentés, dans une dynamique entre auto-catégorisation et catégorisation par autrui (Dubar, 2000). Parallèlement, les travaux de B. Lahire ont montré que, confronté à des univers de socialisation multiples et hétérogènes, l'individu contemporain intègre une pluralité de « répertoires » (ou schèmes de perception et d'action) (Lahire, 2001, 31) qu'il lui revient ensuite d'actualiser, de façon sélective, en fonction des contextes fréquentés. La familiarisation plus ou moins grande à une diversité de contextes sociaux doterait l'individu d'un " sens des situations ", à savoir d'un sens de la pertinence contextuelle de ces répertoires, qui se traduit par la capacité de les mobiliser à bon escient (Lahire, 2001, 42). On trouve des arguments complémentaires dans les travaux de F. de Singly : ce dernier décrit les identités contemporaines comme multidimensionnelles et "à géométrie variable ", faisant l'objet de "réglages identitaires " fré-

1. Les analyses présentées dans cette contribution sont tirées de recherches postdoctorales portant sur le " traitement scolaire de la différence culturelle » en Communauté française de Belgique et en Angleterre, ainsi que les perspectives développées par les élèves « issus de l'immigration » en fin de scolarité obligatoire. Le matériau analysé dans cet article consiste en une cinquantaine d'entretiens socio-biographiques menés à Bruxelles, auprès de jeunes (âgés de 16 à 18 ans), issus de la " seconde génération » des principaux groupes d'immigration significatifs : Marocains, Turcs, Africains (Afrique Centrale surtout). Les entretiens ont été menés à dessein dans des contextes scolaires contrastés (établissements sélectifs, intermédiaires et de « relégation »). Tous accueillent une proportion significative (de 35 à $75 \%$ ) de jeunes " non natifs ». Les entretiens ont été menés selon un principe de faible directivité; trois axes thématiques étaient cependant amenés par nous : la sphère familiale (histoire de la migration familiale, ressources socioculturelles et socioprofessionnelles familiales, attitudes des parents et de la fratrie face à la scolarité), l'expérience scolaire (trajectoire et attentes) et les processus de construction identitaire, au croisement des diverses sphères de socialisation. Pour une présentation approfondie de la méthode, voir Verhoeven, 2005. 
Autrement dit, les positionnements identitaires d'un individu sont liés à sa trajectoire sociale et à la distribution (inégalitaire) des ressources qui y est associée. quents par lesquels l'individu hiérarchise différemment, en fonction des définitions imposées par les situations, les différentes facettes de son identité (de Singly, 2003, p.78-85).

Précisons enfin que cette vision des identités sociales ne se veut ni désocialisée, ni totalement " ouverte $»^{2}$. Le déploiement d'une stratégie identitaire - définie comme l'ensemble des répertoires mobilisés par un individu dans des contextes sociaux différenciés - est en effet inséparable de l'histoire sociale de ce dernier. Autrement dit, les positionnements identitaires d'un individu sont liés à sa trajectoire sociale et à la distribution (inégalitaire) des ressources qui y est associée ${ }^{3}$.

C'est dans cette perspective que cet article analysera les stratégies identitaires déployées par des jeunes "issus de l'immigration ", dans différents contextes scolaires, à partir d'un double questionnement : d'une part, d'un point de vue synchronique, on examinera les répertoires mobilisés par ces jeunes, en particulier les répertoires identitaires (schèmes de perception de soi et de sa propre identité) et les répertoires linguistiques ${ }^{4}$ (pratiques linguistiques renvoyant à des modèles de comportement linguistique socialement disponibles (Hambye, 2005)), en lien avec les différents contextes scolaires (établissements) fréquentés. D’autre part, dans une perspective diachronique, on se penchera sur le rôle de la trajectoire scolaire des jeunes dans la constitution de ces répertoires identitaires et linguistiques.

\section{Stratégies identitaires et contextes scolaires}

L'analyse de récits socio-biographiques menés dans des établissements occupant des positions contrastées sur le "quasi marché scolaire ${ }^{5}$ " met en relief une diversité de stratégies identitaires. Ces stratégies, analysées à partir des répertoires identitaires et linguistiques mobilisés, seront mises en relation avec les contextes scolaires où elles se manifestent de façon privilégiée.

2. Comme pourrait, par exemple, le défendre un point de vue strictement individualiste méthodologique (paradigme du " choix rationnel »), ou encore un constructivisme radical qui insisterait sur le caractère « radicalement toujours ouvert » des contextes de l'interaction au détriment de toute " stabilisation » des ressources socialement héritées.

3. La perspective théorique proposée ici a largement bénéficié des interactions avec les participants au séminaire « Régulation de l'hétérogénéité linguistique en contexte multiculturel. Le français en contact dans les écoles bruxelloises » (convention ARC 0409319 de la Communauté française de Belgique). Je remercie en particulier Jean-Louis Siroux pour ses apports et sa relecture critique.

4. En tant que sociologues, nos compétences actuelles ne nous permettent pas de nous situer clairement dans le champ de la sociolinguistique contemporaine. Signalons toutefois que nos positions semblent rencontrer celles présentées, par exemple, par M. Heller (2002)

5. La notion de " quasi marché ", développée par des chercheurs belges en éducation, renvoie à une régulation du système éducatif articulant une régulation libérale de la demande scolaire (libre choix scolaire pour les parents) et financement public des établissements (lié au nombre d'élèves). Ces caractéristiques contribuent à une forte ségrégation et hiérarchisation des établissements (voir notamment Vandenberghe V., 1997). Dans cet article, nous comparerons des établissements occupant une position privilégiée sur le quasi marché (recrutement socioculturel favorable, peu de retard scolaire, établissement « donneur » d'élèves) avec des établissements occupant une position défavorable (recrutement socioculturel plus défavorisé, retard scolaire plus important, établissement « receveur » d'élèves ayant échoué ailleurs. 


\subsection{Contextes scolaires défavorisés, ethnicisation/essentialisation et hybridation/anomie}

Dans les contextes scolaires défavorisés, les stratégies identitaires observées peuvent être appréhendées à partir de deux répertoires identitaires idéaltypiques ${ }^{6}$ : un répertoire "essentialisant/ethnicisant » et un répertoire "d'hybridation anomique ».

\subsubsection{Le répertoire identitaire essentialiste ou l'héritage non choisi}

Ce premier répertoire se caractérise par une définition essentialiste de l'identité culturelle. Pour ces élèves, la culture d'origine est un héritage non choisi, qui « colle à la peau " et dont on ne peut s'éloigner sans risques. L'appartenance culturelle est vécue de façon totalisante - ces jeunes se disant par exemple "totalement Marocain» ou " $100 \%$ Turc». L'identité officielle et l'histoire vécue en Belgique sont mises à distance : "J'ai peut-être la carte d'identité, mais bon, c'est pas ça qui change ce qu'on a au fond du coeur! Moi, je sens bien que je suis différent, je ne serai jamais

L'analyse de récits socio-biographiques menés dans des établissements occupant des positions contrastées sur le " quasi marché scolaire » met en relief une diversité de stratégies identitaires. Belge" (Mourad, M. ${ }^{7}$ ). Lappartenance à la culture d'origine est vécue comme un socle fondateur essentiel, comme en témoigne ce jeune musulman : "Moi, j’ai des croyances solides, j'ai eu la chance d'avoir des parents qui me les ont transmises très tôt. Pour moi, c'est important qu'on inculque très tôt la culture. Si tu perds ta culture, t'es perdu.» (Shafkat, M.). Toute prise de distance par rapport à la culture d'origine est présentée comme une source de crise personnelle et sociale : "J'en connais, une fois qu'ils renient leur culture, la famille leur dit "OK, tu veux prendre la culture occidentale? Comme tu veux, mais alors nous on ne te veut plus". La famille les rejette complètement. " (Shafkat, M.). L'ancrage communautaire fait ici office de support identitaire (Ehrenberg, 1995), fournissant les repères utiles à la construction du respect de soi dans des univers stigmatisés. On est bien ici dans le cas de figure de "l'ethnicisation de l'exclusion scolaire" pointée par certains sociologues de l'éducation (Debarbieux et alii., 2000, etc.).

\subsubsection{L'anomie ou la nostalgie communautaire}

Un répertoire identitaire idéaltypique inverse, bien que renvoyant à une conception semblable des rapports entre individu et culture, a été identifié. Les jeunes qui s'y réfèrent se présentent comme "ayant perdu leur colonne vertébrale culturelle ", en raison de la mixité des quartiers et du contact avec la culture occidentale, et témoignent d'une sorte de nostalgie de ce socle perdu. Cette élève congolaise explique: "Nous, les Africains, on n'a plus notre culture, on est complètement mélangés, ici. Les Asiatiques de l'école, franchement, je ne les comprends pas : on dirait qu'elles veulent toutes s'européaniser, s'habiller mode et tout. Nous (NDLR: les Africaines) on leur dit: 'Mais enfin, gardez votre culture, profitez-en, quoi! Je crois pas

6. Les positionnements individuels s'échelonnent en une variété de possibles entre ces deux pôles idéaltypiques, qui ne doivent donc en aucun cas être réifiés. La seconde partie de cet article montre d'ailleurs bien à quel point ces répertoires identitaires et linguistiques doivent se comprendre comme une construction dynamique en lien avec des trajectoires sociales et scolaires singulières.

7. Légende des citations : pour contextualiser les entretiens, nous préciserons systématiquement entre parenthèses le prénom (fictif) + le sexe (F ou M). 
qu'elles se rendent compte de la chance qu'elles ont d'avoir encore une culture. Nous, on se sent faibles, perdus. Avoir une culture, ça aide. (...) Nous, les Noirs, on n'a plus de culture, on est juste des Noirs" (Bernadette, F.). Sans socle culturel d'appui à la construction de soi, ces jeunes se retrouvent nus et démunis face à la discrimination. La " différence " se réduit alors au stigmate.

Dans le cadre des répertoires " essentialiste » ou « anomique», la perpétuation de la culture d'origine est présentée comme un atout en termes de construction de soi et d'intégration sociale. En ce sens, on peut dire que ces élèves sont " communautariens ${ }^{{ }}$. Le fait que ces élèves soient favorables à la prise en compte des différences culturelles dans la pédagogie ou la vie des établissements apparaît alors comme une revendication légitime.

\subsubsection{Répertoires linguistiques : une hybridation anomique}

Ces deux répertoires identitaires sont associés à des répertoires linguistiques spécifiques. En ce qui concerne les usages du français, nos observations (en situation scolaire comme en situation d'entretien) mettent en évidence le recours à un français non standard, ponctué de mots d'argot issus du "parler jeune " (Trimaille, 2004) en vigueur dans les quartiers à forte concentration ethnique. Ce "parler jeune » local qui y émerge se caractérise par un mélange de termes empruntés aux langues coprésentes, de mots de la culture jeune et du parler populaire (Back, 1995). Ainsi, cette jeune Marocaine explique fièrement : "J'ai appris un peu de chinois! Oui, parce que j'ai pas mal de potes chinois... J'ai aussi appris quelques mots de turc, et un peu de lingala avec Bernadette, ma copine congolaise. Quand on cause français entre nous, c'est un mélange d'un peu tout. ». Cette " créativité » linguistique s'apparente cependant à un entremêlement des registres et des styles, décrit comme spontané et non réfléchi : "Quand on parle dans la vie de tous les jours, dans la famille, et que par exemple on se marre, ben on va mélanger le français et le berbère. Mais en fait, on réalise pas qu'on mélange, ça se fait tout seul. Il y a des mots qui sortent mieux en français, d'autres mieux en berbère, j'sais pas pourquoi, ça vient comme ça. " Cette hybridation "non réflexive" ou " anomique " des registres semble s'accompagner d'une difficulté à faire preuve d'un "sens des situations " (cf. supra), à savoir d'une difficulté à discerner le registre légitime propre à chaque contexte. Les pratiques linguistiques de ces jeunes sont assez constantes, faisant état d'un "mélange des genres " analogue en situation d'entretien ou en conversation informelle (dans la cour de récréation ou dans la rue). On peut donc faire l'hypothèse que cette hybridation s'apparente à une subculture typique de situations de domination scolaire et sociale (Melliani, 2001).

D'autre part, si une majorité d'élèves affirment fièrement parler leur langue d'origine, ils avouent n'en avoir qu'une connaissance imparfaite, n'ayant pas été alphabétisés dans ces langues. Ceci marque pour eux une rupture dans la filiation et

8. Ils s'appuient en effet sur une conception communautarienne des rapports entre individu et société, au sens de la philosophie politique, puisqu'ils défendent une conception du sujet comme construit à travers son ancrage culturel dans une communauté d’appartenance donnée, et une conception de la citoyenneté et de l'espace public reposant sur la reconnaissance et l'expression libre de cette appartenance. 
suscite des sentiments de honte ou de trahison: " Franchement, je ne suis pas fier. Je me sens mal de pas savoir bien l'arabe, le vrai arabe. C'est surtout par rapport à mes parents, qui sont tristes de voir que je ne suis plus comme eux, et aussi que leurs petitsenfants ne parleront plus leur langue...» (Karim, M.). Le « respect des aînés », souvent brandi comme motif de la maîtrise de la langue d'origine, signe l'ancrage affectif de cette vision: "Quand mes grands-parents me parlent Punjabi, je réponds en «bon Punjabi ». Je vais pas mettre des mots d'argot. Pareil avec mon père. C'est une question de respect : je dois leur parler comme il faut. » (Shafkat, M.).

\subsection{Contextes scolaires favorables et stratégie assimilationniste}

Dans les établissements en position favorable, certains jeunes font état d'une stratégie assimilationniste.

\subsubsection{Assimilation culturelle revendiquée et mise à distance de l'origine}

Ce répertoire identitaire consiste en la revendication affirmée d'une appartenance forte, voire exclusive, au pays d'accueil, accompagnée d'un rejet systématique de toute appropriation d'une prétendue « origine». Ceci passe par un appui sur la dimension officielle de l'identité ("Vous savez, j'ai la carte d'identité belge!») qui vient légitimer un sentiment d'appartenance ("Je suis aussi Belge que vous, hein! Moi, je ne me suis jamais sentie "Marocaine - Marocaine"! (Farida, F.). Ceci s'accompagne de l'appropriation subjective de marqueurs culturels du contexte d'accueil : "Vous savez, moi je mange des steak-frites, hein, je suis un enfant du Nord!" (Karim, M.); "Personnellement, je n'aime pas du tout la musique traditionnelle de mes parents; moi, ce que j'aime, c'est le pop français. » (Wassila, F). Ces élèves sont plutôt « libertariens » et égalitaristes : être citoyens légitimes d'un espace public suppose pour eux d'être considérés comme individus égaux (au sens moderne et abstrait), et non comme membres d'une communauté particulière. Ainsi, ils refuseront que les enseignantes et les enseignants s'appuient sur les particularités culturelles ou religieuses pour construire les programmes, préférant être « traités comme les autres ».

\subsubsection{Répertoires linguistiques : maîtrise du français standard et rejet des langues d'origine}

Parallèlement, le répertoire linguistique assimilationniste articule deux aspects. D’une part, les élèves adoptent aisément les normes du français standard : leur construction grammaticale et leur niveau de vocabulaire sont très corrects et on n'y décèle quasiment pas de mots qui pourraient s'apparenter à un "parler jeune ». Ils recourent aisément aux codes que requiert la situation d'entretien. Bons élèves, ils sont capables de répondre aux exigences de la langue scolaire. D'autre part, ils ne semblent pas souhaiter que les langues d'origine soient étudiées à l'école. Deux cas se sont présentés. Certains disent méconnaître ces langues : "Moi, l'arabe, je ne le parle pas bien. Mes grands-parents me parlent arabe mais j'ai toujours répondu en français; ça me gêne un peu de parler arabe, parce que je sais bien que j'ai un accent et que je fais des fautes. " D'autres disent parler la langue d'origine, au même titre que le français et parfois que d'autres langues de contact (en cas de trajectoire migratoire 
« à rebondissement ${ }^{9}$ »). Cependant, ce bi- (ou multi-) linguisme n'est pas mobilisé sur la scène scolaire. Interrogés sur leur souhait de suivre des cours de langue d'origine à l'école, leur réponse est claire : « Non! Et même s'il y avait eu cette possibilité, je n'aurais pas pris.(...) Pour moi, si on veut parler turc, il y a la maison. A l'école, c'est bien d'avoir des bons cours de français, ou alors des cours d'anglais, de néerlandais. " (Okmès, M.). L'entrée des langues de la migration dans l'espace scolaire n'est pas légitime. La clé de l'intégration passe par la maîtrise de la langue d'accueil.

\subsection{Contextes scolaires favorables et stratégies identitaires complexes}

D'autres jeunes scolarisés dans des établissements performants font état de stratégies identitaires complexes.

\subsubsection{Hybridation réflexive et diffraction identitaire stratégique}

Deux répertoires identitaires idéaltypiques ont été identifiés ici : l'« hybridation réflexive" et la "diffraction identitaire stratégique». Le premier s'apparente au " métissage " défini par M. Wiewiorka, à savoir à un "processus individuel créatif " d'exploration des mélanges à partir de la rencontre de deux ou plusieurs univers culturels, qui permet au sujet individuel de se dire, de se "traduire en actes" (Wieviorka, 2001 : 75). Ce "mélange des codes" fait l'objet d'un travail réflexif assumé. Le second répertoire renvoie à un usage stratégique et différencié de codes culturels différents, en fonction des contextes d'action. Autrement dit, si le premier répertoire relève de la composition personnelle, le second s'apparente davantage à un sens stratégique des situations (cf. supra).

Dans le premier cas, les jeunes présentent une image plurielle d'eux-mêmes (" un peu Arabe, un peu Bruxelloise... »), et mettent en avant leur façon « bien à eux " de combiner différents héritages. L'essentiel n'est pas ici les contenus culturels mobilisés (puisés indifféremment dans les différents mondes fréquentés), mais la mise en avant d'un travail autonome d'analyse, de tri et de réappropriation subjective de ceux-ci. Ainsi, W. explique qu'il ne se sent " ni Belge, ni Indien, mais tout simplement moi-même, c'est-à-dire, un fameux mélange!». Prenant l'exemple de sa façon de vivre sa vie amoureuse, il dit avoir fait siennes certaines des valeurs de la culture familiale - telles les restrictions dans les relations intimes avant le mariage tout en s'en éloignant d'autres (il a choisi une jeune fille d'une autre origine culturelle que lui). "Il y a des choses auxquelles je crois, que je veux garder; mais en même temps, sur qui je choisis d'aimer, là-dessus, je ne veux pas céder... " (Welayat, M.). Cette attitude apparaît également à propos de questions religieuses, comme chez ces jeunes musulmanes qui déclarent que c'est à la suite de démarches de lecture et de réflexion philosophique qu'elles projettent de porter le voile. "J'ai beaucoup lu, beaucoup réfléchi, j'ai discuté avec des tas de gens; si je décide de le mettre, ce ne sera pas comme

9. Nous faisons ici référence à des trajectoires migratoires familiales ayant impliqué des séjours plus ou moins prolongés dans différents pays, pour diverses raisons (par exemple, le séjour dans un premier pays d'émigration est parfois la première étape, nécessaire politiquement ou administrativement, pour en atteindre un second; ou encore, l'installation dans un premier pays d'accueil est entravée par de nouveaux problèmes économiques ou politiques, et suivie d'un nouveau départ, etc.). 
ma mère, juste parce que c'est comme ça (...). Moi j'ai fait tout un cheminement pour arriver à cette décision" (Samira, F.). Ces jeunes font ainsi preuve d'une capacité à « faire le tri dans leurs héritages » et à « décider du poids du passé qu'ils souhaitent incorporer " (de Singly, 2003 : 30-31). Le second répertoire - la "diffraction identitaire stratégique " - renvoie à une attitude stratégique et contextualisée : il s'agit d'élèves qui, tout en assumant la pluralité des influences culturelles qui les forgent, choisissent de les adopter successivement, en fonction des sphères de vie. Ces jeunes diront « jouer leur rôle » de jeune Turc, Marocain ou Pakistanais, dans certains espaces (la famille, la communauté), et adopter une tout autre présentation de soi dans d'autres lieux (l'école, la rue). "Je ne peux pas me permettre d'être pareil partout, c'est simple... En famille, par exemple, je serai très taiseux, je rentre chez moi, je m'assieds, je lis le journal... À l'école, c'est tout le contraire, on dirait qu'il faut que je me défoule, je suis hyper bavard, je participe beaucoup au cours..." (Okmès, M.). Les jeunes qui recourent à ce répertoire font ainsi preuve d'un grand "sens des situations ", dans la mesure où leur identité semble présenter une forte malléabilité en fonction des profits ou des sanctions auxquels pourraient les exposer telle ou telle posture dans tel contexte social. Cette façon de gérer l'identité dans un monde pluriel se rapproche de la notion d'« identité à géométrie variable» (de Singly, 2003, p. 78).

\subsubsection{Usage de codes linguistiques différenciés et capacité de " crossing "}

Tout comme les "assimilés ", les élèves adoptant ces deux derniers registres identitaires se révèlent être des utilisateurs avertis du français standard. Les entretiens ne comportent que très peu de mots d'argot ou de "parler jeune ${ }^{10}$ - qu'ils utiliseront pourtant lors de conversations informelles avec des pairs (observées dans le quartier, par exemple), car ils sont conscients des moments où ce langage peut être utilisé sans appeler de sanction sociale ou scolaire. Parallèlement, les langues d'origine sont valorisées. Beaucoup suivent des cours dispensés dans des associations communautaires et disent souhaiter une maîtrise plus fine de la langue et un accès à la culture grâce à l'écrit. Certains élèves vont jusqu'à suivre des cours d'autres langues minoritaires qu'ils côtoient. Le bi- et le multi-linguisme sont valorisés, comme chez cet élève d'origine turque et albanaise qui affirme fièrement parler six langues, suite à une histoire migratoire à rebondissements, et espère en tirer profit pour son insertion : «Oui, moi je suis sûr que ça va me servir plus tard; j'aimerais bien travailler comme interprète, ou comme médiateur culturel; j'ai déjà pensé faire ça dans le milieu hospitalier, et je sais qu'on en cherche. Là, j'ai des atouts...» (Orin, M.). Bref, ces élèves tiennent un discours réflexif sur les usages pertinents des répertoires linguistiques - qu'il s'agisse du français, de la langue d'origine ou du «parler jeune ». Loin de «mélanger les genres ", ils les utilisent à bon escient. L'hybridation se définit alors comme utilisation ad hoc et réflexive de langues et de registres qui s'apparente $\mathrm{au}$ « crossing » des sociolinguistes (Rampton, 1995).

10. Lorsqu'ils y recourent, c'est toujours avec l'humour ou la « distance " qui marquent la conscience de la nonadéquation du terme par rapport au registre discursif attendu. 


\section{Une approche diachronique : construction des répertoires et trajectoires scolaires}

L'analyse synchronique peut maintenant être complétée par une approche plus dynamique, consistant en une analyse diachronique du rôle de la trajectoire scolaire dans la construction de ces répertoires. Chaque trajectoire singulière peut être lue comme le cumul des socialisations successives dans des établissements contrastés, qui proposent des cadres d'appréhension différents de la différence culturelle autorisant ainsi un espace différentiel des possibles aux stratégies identitaires.

\subsection{Des logiques d'établissements}

Nos travaux (Verhoeven, 2003) ont mis en évidence que, dans des systèmes scolaires de " quasi-marché » (cf. noteV), plus les établissements occupent une position dominante, plus ils tendent à promouvoir une logique individualiste et méritocratique peu compatible avec la reconnaissance scolaire des appartenances culturelles; la définition des rapports enseignants - enseignés s'y construit sur un mode " rationneluniversalisant" détaché de l'expression des affects et des particularismes. À l'inverse, plus on «descend» dans la hiérarchie entre établissements, plus ceux-ci tendent à valoriser la prise en compte des particularismes et des parcours singuliers.

\subsection{Attentes normatives des établissements et élaboration diachronique des répertoires}

L'analyse des récits montre combien le processus de construction des répertoires des élèves est étroitement lié aux attentes des établissements successivement fréquentés - les répertoires des jeunes s'y voyant soit confortés, soit découragés.

\subsubsection{Stratégies essentialistes, ghettoïsation ou mobilité descendante}

Les élèves qui déploient des stratégies essentialistes se caractérisent souvent par des histoires scolaires peu mobiles, voire " ghettoïsées » : au cours d'une socialisation concentrée sur un même territoire - celui de quartiers « à forte densité immigrée »-, ils ont peu été confrontés à d'autres normes que celles de cette subculture. Insuffisamment familiarisés aux normes dominantes, ils semblent peu à même de s'y conformer. Plus souvent, l'ethnicisation correspond à une stratégie de repli, associée à une trajectoire scolaire descendante. Chaouki fait partie de ces jeunes qui, bons élèves en primaire, se retrouvent confrontés, à l'entrée du secondaire, à des établissements sélectifs (suite aux stratégies des parents ou d'un(e) enseignant(e)). Il ne résistera pas aux pressions de cet univers, non pas tant sur le plan scolaire, mais sur le plan de la stigmatisation dont il y fera l'objet («En première, on était trois Marocains, encaqués au fond de la classe près du radiateur. C'est dans cette école que j'ai entendu pour la première fois l'expression "Sale Marocain »...) et des transactions identitaires attendues, dans un établissement qui exige qu'on "laisse sa culture au vestiaire». Il optera vite pour une école moins performante mais plus ouverte à la différence («Ici, je me suis tout de suite senti accepté comme personne, tel que je suis, et pas pour ce que je pourrais devenir $»$. 


\subsubsection{L'hybridation non réflexive : trajectoires nomades et mobilité inversée}

L'« hybridation non réflexive" s'observe le plus souvent chez des élèves de familles au capital socioculturel peu élevé, qui ont vécu une trajectoire migratoire à rebondissements (cf. note IX), mais où cette mobilité a été vécue négativement par le jeune, comme un « brouillage de repères ». Arrivés en Belgique, leur scolarisation est chaotique. Ainsi, Neema, arrivée à Bruxelles suite aux troubles politiques dans son pays (Rwanda), passe un an en classe de «primo arrivants » et y apprend le français aux côtés de jeunes de douze nationalités différentes. Elle tente ensuite une première dans un établissement favorisé, avant d'échouer et de se retrouver dans un établissement " multiethnique » du quartier, moins bien positionné. Il semble que de telles trajectoires biographiques, à la fois socialement chaotiques et scolairement « descendantes », ne permettent aux élèves ni d'accéder aux ressources nécessaires pour décoder les situations scolaires "dominantes", ni de développer efficacement un "sens des situations" (ce qui demande, en fait, une familiarisation suffisante et " ordonnée " à une pluralité de contextes sociaux). On peut faire l'hypothèse que l'hybridation qui se produit dans ces cas-là est peu capitalisable dans le champ scolaire (ce qui n'enlève rien à son éventuelle valeur identitaire auprès des groupes de pairs, par exemple).

\subsubsection{Stratégies assimilationnistes : ascension scolaire ou trajectoires dominantes-résistantes}

Deux types de trajectoires scolaires contribuant à la consolidation d'un répertoire assimilationniste ont été observés. Dans certains cas, il s'agit d'élèves en mobilité sociale et scolaire ascendante, qui se conforment progressivement aux attentes des établissements bien positionnés. Ainsi, Rachida, scolarisée dans la filière " noble » d'un établissement moyen, explique qu'elle a commencé sa scolarité dans un établissement de quartier, multiethnique et "familial: c'était l'ambiance qui comptait, les profs nous connaissaient tous... ». Elle est parvenue à s'appuyer sur les ressources offertes dans cet établissement (notamment en termes de reconnaissance culturelle) pour réussir : "Je me sentais respectée pour ce que j'étais, et en même temps j'ai vite compris que si je voulais m'en sortir, il faudrait que je sois meilleure que tout le monde. (...) Monsieur D., le prof de français, il a cru en moi et il a persuadé mes parents qu'il fallait que j'aille dans une bonne école.» (Rachida, F.). Dans d'autres cas, il s'agit d'élèves issus de milieux plus aisés, que les parents, aptes à décoder les mécanismes de sélection scolaire, ont inscrits dans des établissements sélectifs. Ainsi, Soufiane, fils d'universitaires marocains, raconte: "Si on a pu rentrer à l'Athénée A (établissement élitiste bruxellois), c'est parce que maman a fait le forcing, et qu'elle a refusé qu'on lui dise gentiment qu'il n'y avait plus de place. » Pour « tenir le coup ", il s'agit pour ces élèves de mener en permanence une double stratégie de présentation de soi comme " assimilé » (« Montrer les signes d'appartenance à l'école? Surtout pas! Ce serait leur mettre le pied à l'étrier! - De quoi? - ben, de la discrimination...») et de performance scolaire irréprochable ("Si on a tenu le coup, c'est que nos parents nous ont répété, depuis qu'on était tout petits : "Toi, comme tu es Marocain, tu n'as pas le droit à l'erreur; à la moindre erreur, ils t'auront, tu dois être meilleur que les 
autres si tu veux réussir " (Soufiane, M.). L'accès à des ressources familiales ou scolaires (l'enseignant(e) ou le parent « averti » des mécanismes de la sélection) joue un rôle crucial dans l'accrochage à ce type d'univers scolaire.

\subsubsection{Stratégies complexes et incitants scolaires à jongler avec plusieurs mondes culturels}

Enfin, quelles trajectoires scolaires caractérisent les élèves qui développent des stratégies identitaires complexes? Il s'agit souvent, ici encore, d'élèves aux capitaux socioculturels relativement élevés, et dont la trajectoire familiale et scolaire a permis non seulement de se familiariser aux codes scolaires dominants, mais aussi de cultiver un "sens des situations ». L'adoption de stratégies non assimilationnistes semble aussi tenir à la rencontre d'univers - ou d'acteurs - scolaires qui, à un moment ou l'autre du cursus, légitiment la diversité culturelle et facilitent l'apprentissage à « jongler avec plusieurs mondes ». Ainsi, les récits de ces élèves font souvent état du soutien de l'un ou l'autre enseignant ou de l'une ou l'autre enseignante, qui a réussi, à partir d'un rôle de médiation entre l'école et la famille, à légitimer les valeurs de l'une aux yeux de l'autre et réciproquement (parvenant ainsi à éviter la "double distance" culturelle expérimentée par les jeunes de l'immigration et à favoriser une "schizophrénie heureuse " (Lahire, 1998)). Dans d'autres cas, ce qui s'avère décisif, c'est la fréquentation d'un «bon » établissement scolaire conciliant l'excellence scolaire avec une politique de la différence respectueuse de la diversité. Il s'agit souvent de bons établissements qui, confrontés à un public de plus en plus diversifié culturellement, ont progressivement pris leurs distances par rapport à une philosophie « universaliste » et opté pour une conception « cosmopolite » du rapport aux cultures. En rendant légitime la diversité culturelle, l'institution scolaire ou ses acteurs permettent aux élèves de " capitaliser » leurs répertoires au sein de l'espace scolaire. Ceux-ci ne se sentent plus tiraillés entre des identités irréconciliables, mais autorisés à partir de leurs expériences multiples à construire un rapport critique à toutes les identités.

\section{Conclusion et perspectives}

\subsection{Théorie sociologique des identités et institution scolaire}

Ce cheminement nous permet d'étayer notre souci de dépassement des théories classiques de l'intégration scolaire des jeunes de minorités. 


\begin{tabular}{|l|l|l|}
\hline $\begin{array}{l}\text { Rapport Position scolaire } \\
\text { à la culture } \\
\text { et à la langue }\end{array}$ & $\begin{array}{l}\text { Position scolaire } \\
\text { valorisée / trajectoire } \\
\text { ascendante }\end{array}$ & $\begin{array}{l}\text { Position scolaire } \\
\text { défavorable / trajectoire } \\
\text { descendante }\end{array}$ \\
\hline $\begin{array}{l}\text { Rapport univoque et } \\
\text { totalisant à l'identité, à la } \\
\text { culture et à la langue }\end{array}$ & $\begin{array}{l}\text { Assimilationnisme culturel } \\
\text { // Maîtrise du français } \\
\text { dominant et rejet des } \\
\text { langues d'origine }\end{array}$ & $\begin{array}{l}\text { Essentialisation } \\
\text { // Faible maîtrise des } \\
\text { différentes langues et } \\
\text { des registres discursifs } / / \\
\text { Hybridation langagière } \\
\text { comme « subculture » }\end{array}$ \\
\hline $\begin{array}{l}\text { Rapport flexible et complexe } \\
\text { aux identités et aux langues }\end{array}$ & $\begin{array}{l}\text { Hybridation réflexive et } \\
\text { diffraction stratégique } \\
\text { // Usage réflexif et ad hoc } \\
\text { des répertoires linguistiques }\end{array}$ & $\begin{array}{l}\text { Hybridation anomique } \\
\text { // Hybridation langagière } \\
\text { comme « subculture » }\end{array}$ \\
\hline
\end{tabular}

$\mathrm{Au}$ couple conceptuel classique "assimilation - essentialisation", vient se superposer un second axe, opposant les stratégies de " flexibilité identitaire " (l'hybridation réflexive et la diffraction stratégique, figures dominantes de la capacité à vivre dans plusieurs mondes), à l' "hybridation anomique ", définie comme difficulté subjective à gérer réflexivement la pluralité de référents. In fine, l'essentialisation et l'anomie peuvent être lues comme les deux faces de cette même difficulté à se construire dans un monde pluriel : l'une correspond à l'éclatement ou à "l'implosion », l'autre à la « crispation identitaire » (Kaufmann, 2004).

\subsection{Des formes dominantes et dominées de multilinguisme}

Parallèlement, sur le plan sociolinguistique, ce second axe renvoie à deux formes irréductibles de rapport à la diversité linguistique : dans un cas, la diversité des répertoires linguistiques côtoyés s'apparente davantage à une subculture (caractérisée par une hybridation non réflexive et un non- discernement des usages sociaux des langues et des registres); dans l'autre, il s'agit au contraire d'une compétence éminemment " gagnante » dans le monde contemporain : la capacité à utiliser de façon performante plusieurs langues et plusieurs registres de langues de façon adéquate aux attentes normatives des contextes.

\section{Références bibliographiques}

BARRERE, Anne et SEMPEL, Nicolas (1998). Sociologie de l'éducation. Paris : Nathan Université.

BACK, Les (1995). New Ethnicities and Urban Culture. Racism and Multiculture in Young Lives. London : UCL Press. 
DEBARBIEUX, Éric, GARNIER, Alix et MONTOYA, Yves (2000). La violence en milieu scolaire. 2, Le désordre des choses. Paris : ESF, coll. Actions sociales/ confrontations.

DUBAR, Claude (2000). La crise des identités. L'interprétation d'une mutation. Paris : Presses universitaires de France.

EHRENBERG, Alain (1995). L'individu incertain. Paris : Calmann-Lévy, coll. Essai Société.

GELLNER, Ernest (1994). Nations et nationalismes. Paris : Payot.

HAMMERSLEY, Martin et WOODS, Peter (ED.) (1973). The Process of Schooling : a Sociological Reader. London : Routledge, coll. The Open University.

HAMBYE, Philippe (2005). La prononciation du français contemporain en Belgique. Variation, normes et identité. Thèse de doctorat (à paraître), Ottignies Louvain-la-Neuve : Université catholique de Louvain-La-Neuve.

HELLER, M. (2002). Éléments d'une sociolinguistique critique. Paris : Éd. Didier, coll. Langues et apprentissage des langues.

KAUFMANN, Jean-Claude (2004). L'invention de soi. Une théorie de l'identité. Paris : Armand Colin.

LAHIRE, Bernard (1998). La réussite scolaire en milieux populaires ou les conditions d'une schizophrénie heureuse. Ville-École-Formation (migrantsFormation), $\mathrm{n}^{\circ}$ 114, septembre, pp. 104-109.

LAHIRE, Bernard (2001). L'homme pluriel : les ressorts de l'action. Paris : Nathan, coll. Essais et recherches, série sciences sociales.

MELLIANI, F. (2001). Subculture et territorialités urbaines en banlieue rouennaise. Cahiers de Sociolinguistique, $\mathrm{n}^{\circ}$ 6, pp. 64-74.

RAMPTON, B. (1995). Crossing : Language and Ethnicity among Adolescents. London - New York : Longman.

SINGLY, François de (2003). Les uns avec les autres. Quand l'individualisme crée du lien. Paris : Armand Colin.

TRIMAILLE, C. (2004). Études de parlers de jeunes urbains en France. Éléments pour un état des lieux. Cahiers de sociolinguistique, nº 9, pp. 99-132.

VANDENBERGHE, Vincent (1997). Le dispositif institutionnel scolaire belge : un mécanisme hybride alliant l'état et le marché, marqué par la ségrégation entre écoles. Savoir, 9/ 3-4, pp. 363-375.

VERHOEVEN, Marie (1997). Les mutations de l'ordre scolaire. Régulation et socialisation dans quatre établissements scolaires contrastés.

Louvain-la-Neuve : Académia-Bruylandt. 
VERHOEVEN, Marie (2003). Modèles d'intégration nationaux, dynamiques d'établissements et processus identitaires en contextes multiculturels : regards croisés Angleterre - Communauté française de Belgique. Revue Française de Pédagogie, nº 144, Juillet-Août-Septembre, pp. 9-17.

VERHOEVEN, Marie (2005). Traitement scolaire de la différence culturelle et identités de jeunes issus de l'immigration. Une recherche semi-inductive et comparative multi-niveaux, dans Vers des critères de qualité pour la recherche qualitative en éducation. Hommage à Michaël Huberman, sous la direction de L. Paquay. Bruxelles : De Boeck (sous presse). 